\title{
The role of individual intertemporal transfers in dynamic TU-Games
}

Citation for published version (APA):

Berden, C. (2007). The role of individual intertemporal transfers in dynamic TU-Games. METEOR, Maastricht University School of Business and Economics. METEOR Research Memorandum No. 030 https://doi.org/10.26481/umamet.2007030

Document status and date:

Published: 01/01/2007

DOI:

10.26481/umamet.2007030

Document Version:

Publisher's PDF, also known as Version of record

\section{Please check the document version of this publication:}

- A submitted manuscript is the version of the article upon submission and before peer-review. There can be important differences between the submitted version and the official published version of record.

People interested in the research are advised to contact the author for the final version of the publication, or visit the DOI to the publisher's website.

- The final author version and the galley proof are versions of the publication after peer review.

- The final published version features the final layout of the paper including the volume, issue and page numbers.

Link to publication

\footnotetext{
General rights rights.

- You may freely distribute the URL identifying the publication in the public portal. please follow below link for the End User Agreement:

www.umlib.nl/taverne-license

Take down policy

If you believe that this document breaches copyright please contact us at:

repository@maastrichtuniversity.nl

providing details and we will investigate your claim.
}

Copyright and moral rights for the publications made accessible in the public portal are retained by the authors and/or other copyright owners and it is a condition of accessing publications that users recognise and abide by the legal requirements associated with these

- Users may download and print one copy of any publication from the public portal for the purpose of private study or research.

- You may not further distribute the material or use it for any profit-making activity or commercial gain

If the publication is distributed under the terms of Article $25 \mathrm{fa}$ of the Dutch Copyright Act, indicated by the "Taverne" license above, 


\section{Caroline Berden}

The Role of Individual Intertemporal Transfers in Dynamic TU-Games

$\mathrm{RM} / 07 / 030$

JEL code: C71, C73

\section{METEबR

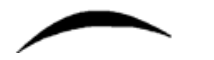

Maastricht research school of Economics

of TEchnology and ORganizations

Universiteit Maastricht

Faculty of Economics and Business Administration

P.O. Box 616

NL - 6200 MD Maastricht

phone : ++31433883830

fax $\quad$ : ++31433884873 



\title{
The Role of Individual Intertemporal Transfers in Dynamic TU-Games*
}

\author{
Caroline Berden ${ }^{\dagger}$
}

July 30, 2007

\begin{abstract}
Dynamic TU-games are considered which consist of a finite player set, a finite sequence of TU-games and a profile of intertemporal utility functions. At every stage a (restrictively) additive solution is applied to the TU-game, which results in a stream of payoff distributions, evaluated by the intertemporal utility functions of the players. Players are able to transfer payoffs between stages. The strategic possibilities from individual transfers between periods are modeled by a noncooperative game. Conditions under which a Nash equilibrium in this noncooperative game exists, are established. It is shown when a Nash equilibrium in dominant strategies is Pareto optimal.
\end{abstract}

Keywords: Cooperative games, Dynamic games.

JEL Codes: C71, C73

\section{Introduction}

A cooperative game with transferable utility is characterized by a function which gives each coalition of players a nonnegative worth. This game describes a one-time event. However, in reality events happen repeatedly over time or are related to earlier events, e.g. treaty and contract negotiations. This is captured by a dynamic cooperative game with transferable utility, where at every stage a cooperative game with transferable utility is played. Furthermore, every player has a utility function to enable him to evaluate a stream of payoff distributions. A solution is applied to the dynamic game and gives a (set of) payoff distribution(s) for every time period. In this paper, additive and restrictively additive solutions are considered. The well-known Shapley value (Shapley, 1953) is an example of

*The author would like to thank Hans Peters for helpful comments.

${ }^{\dagger}$ Department of Quantitative Economics, University of Maastricht, P.O. Box 616, 6200 MD Maastricht, The Netherlands. Tel.: +31 43 3883835, email: c.berden@ke.unimaas.nl 
a (restrictively) additive solution. The nucleolus (Schmeidler, 1969) and prenucleolus are restrictively additive solutions which are explored in more detail.

Some assumptions are made about the dynamic game. First, the player set remains the same in every stage game within the dynamic cooperative game. Another assumption is that the sequence of stage games is exogenously determined. The time horizon is assumed to be finite and time is considered to be discrete. ${ }^{1}$ Particular intertemporal utility functions are considered that value time periods closer to the present more than time periods further away from the present. An example is discounted utility (Fishburn et al., 1982), which is well-known in literature and often used in economics.

In real life situations events are often history dependent. This aspect can be modeled in a dynamic game by letting the stage games and/or solutions over these stage games depend on the history of play. Filar and Petrosjan (2000) consider dynamic games in which the stage games depend on payoffs that are obtained in earlier periods. In particular they focus on solutions which are time consistent to the stage games. The present paper is based on Kranich et al. (2000) who assume that the sequence of stage games is given. The authors develop theoretical tools to analyze dynamic games and discuss the possibility of borrowing and saving over time by introducing individual and aggregate transfers. Although the sequence of stage games before any payoffs are transferred is exogenously determined, transfers change the stage games endogenously.

The idea of borrowing and saving is implemented in the model described in this paper and players are allowed to individually transfer payoffs between stage games within the dynamic game. The following two transfer systems are considered. In the first system an individual transfer only influences the individual worths of stage games within a dynamic game. In contrast, in the second system an individual transfer also influences all worths of coalitions over time which contain the individual. We make the assumption that the change of a coalition's worth equals the sum of the changes of the individual worths of players contained in the coalition. To ensure that the worths of coalitions remain nonnegative under the second transfer system, only dynamic cooperative games with weakly superadditive stage games are considered. In a weakly superadditive game the worth of a coalition is at least equal to the sum of the individual worths of individuals contained in the coalition. The strategic possibilities which result from individual intertemporal payoff transfers are modeled by a noncooperative game, which depends on the underlying dynamic cooperative game, a solution concept and a transfer system.

The paper is concerned with finding conditions under which a Nash equilibrium exists in the above described noncooperative game. In Theorem 4 existence is proven for both transfer systems in case the solution is continuous and transfer- $k$-concave and the intertemporal utility functions of the dynamic game are continuous, increasing and quasi-concave. These assumptions ensure that the utility functions of the associated noncooperative game are continuous and quasi-concave, which are two of the sufficient conditions under which

\footnotetext{
${ }^{1}$ The above mentioned assumptions are also made in a paper by Kranich, et al. (2005) in which different core concepts for dynamic games with transferable utility are considered.
} 
Debreu (1952) proved existence of a Nash equilibrium in a noncooperative game. Example 4 illustrates that a Nash equilibrium does not have to exist in case the nucleolus is applied to a dynamic cooperative game where payoffs are transferred according to the first transfer system. This is consistent with the fact that the nucleolus is not transfer-1-concave, i.e. the nucleolus is not concave under the first transfer system. Therefore not all utility functions of the associated noncooperative game are quasi-concave. Hence, transfer- $k$-concavity of the solution cannot be dropped from the theorem. Additionally, it is shown that a Nash equilibrium in dominant strategies exists if the intertemporal utility functions of the dynamic game value the present more than the future and one of two instances are met: (i) payoffs are transferred according to the first transfer system, the solution is additive and some other specific conditions on the solution hold or (ii) payoffs are transferred according to the second transfer system, the dynamic game only consists of weakly superadditive cooperative games, the solution is restrictively additive and another specific condition on the solution hold. In this special type of Nash equilibrium players transfer as much payoff as possible to the present. Under particular conditions this Nash equilibrium is shown to be Pareto optimal.

The organization of the paper is as follows. Section 2 presents the model and main definitions. Section 3 presents the main results. Section 4 is devoted to Pareto optimality. Section 5 concludes.

\section{Preliminaries}

A game with transferable utility (TU-game) consists of a pair $(N, v)$ where $N:=\{1, \ldots, n\}$ denotes the set of players and $v: 2^{N} \rightarrow \mathbb{R}$ is a characteristic function which assigns to each coalition $S \subseteq N$ the worth $v(S)$, such that $v(S) \geq 0$ for all $S \subseteq N$ and $v(\emptyset)=0$. In the remainder we omit $N$ from notation and simply write $v$. The set of all TU-games with player set $N$ is denoted by $\mathcal{G}^{N}$. A game $v \in \mathcal{G}^{N}$ is additive if $v(S \cup T)=v(S)+v(T)$ for all disjoint $S, T \subseteq N$. Hence, the additive game $v \in \mathcal{G}^{N}$ is generated by the vector $(v(1), \ldots, v(n)) \in \mathbb{R}^{N}$. A game $v \in \mathcal{G}^{N}$ is weakly superadditive in case $v(S) \geq \sum_{i \in S} v(i)$ for all $S \subseteq N$. The zero-game is denoted by $z \in \mathcal{G}^{N}$.

Definition 1: Let $T \in \mathbb{N}$. A dynamic $T U$-game $\Gamma$ is a pair $(\mathbf{v}, \mathbf{u})$ where

(i) $\mathbf{v}=\left(v^{1}, \ldots, v^{T}\right) \in\left(\mathcal{G}^{N}\right)^{T}$.

(ii) $\mathbf{u}=\left(u_{1}, \ldots, u_{n}\right)$ where, for each $i \in N, u_{i}: \mathbb{R}^{T} \rightarrow \mathbb{R}$.

The function $u_{i}$ assigns to every payoff stream $\mathbf{x} \in \mathbb{R}^{T}$ of player $i$ utility level $u_{i}(\mathbf{x})$. If for all $\mathbf{x}, \mathbf{y} \in \mathbb{R}^{T}$ with $\mathbf{x}>\mathbf{y}^{2}$ it holds that $u_{i}(\mathbf{x}) \geq u_{i}(\mathbf{y})\left[u_{i}(\mathbf{x})>u_{i}(\mathbf{y})\right]$, then $u_{i}$ is [strictly] increasing. The function $u_{i}$ is quasi-concave if for all $\mathbf{x}, \mathbf{y} \in \mathbb{R}^{T}$ and $\lambda \in(0,1)$ it holds that $u_{i}(\lambda \mathbf{x}+(1-\lambda) \mathbf{y}) \geq \min \left\{u_{i}(\mathbf{x}), u_{i}(\mathbf{y})\right\}$.

\footnotetext{
${ }^{2}$ The vector inequality $\mathbf{x}>\mathbf{y}$ is to be understood as $x^{t} \geq y^{t}$ for all $t \in\{1, \ldots, T\}$ and $x^{t}>y^{t}$ for at least one $t \in\{1, \ldots, T\}$.
} 
In order to define a special class of intertemporal utility functions, the vector $\mathbf{e}^{t} \in \mathbb{R}^{T}$ is defined which has a 1 for the element on place $t$ and zeros for all other elements.

Definition 2: The class $\mathcal{V}[$ class $\overline{\mathcal{V}}]$ contains intertemporal utility functions $u: \mathbb{R}^{T} \rightarrow \mathbb{R}$ with the property that for all $\mathbf{x}, \mathbf{y} \in \mathbb{R}^{T}$, all $t, t^{\prime} \in\{1, \ldots, T\}$ and $\epsilon>0$ it holds that if $t<t^{\prime}$ and $\mathbf{x}=\mathbf{y}+\epsilon \mathbf{e}^{t}-\epsilon \mathbf{e}^{t^{\prime}}$, then $u(\mathbf{x}) \geq u(\mathbf{y})[u(\mathbf{x})>u(\mathbf{y})]$.

Clearly, $\overline{\mathcal{V}} \subset \mathcal{V}$. The well-known and in economics often used class of discounted utility is contained in the class $\mathcal{V}$. According to discounted utility, the utility level of a payoff in a payoff stream when received immediately is multiplied with a discount factor that corresponds to the time of receival of the payoff. This procedure is repeated for every payoff within the stream and generates discounted payoffs. All these discounted payoffs are summed in order to obtain the level of discounted utility of a payoff stream. The next lemma characterizes differentiable utility functions in $\mathcal{V}$.

Lemma 1 For all utility functions $u: \mathbb{R}^{T} \rightarrow \mathbb{R}$ for which the partial derivatives exist and are continuous and $u_{t}^{\prime}(\mathbf{x}) \geq 0{ }^{3}$ for all $t$ and all $\mathbf{x} \in \mathbb{R}^{T}$, we have $u \in \mathcal{V}$ if and only if $u_{t}^{\prime}(\mathbf{x}) \geq u_{t^{\prime}}^{\prime}(\mathbf{x})$ for every $\mathbf{x} \in \mathbb{R}^{T}$ and all $t<t^{\prime}$.

Proof. Let $t<t^{\prime}$ and $\mathbf{y}_{\eta}:=\mathbf{y}+\eta \mathbf{e}^{t}-\eta \mathbf{e}^{t^{\prime}}$ for all $\mathbf{y} \in \mathbb{R}^{T}$ and $\eta>0$. Let $\epsilon>0$ and define the function $f:[0, \epsilon] \rightarrow \mathbb{R}$ by $f: \eta \mapsto u\left(\mathbf{y}_{\eta}\right)$. Then, using the chain rule,

$$
\begin{aligned}
u\left(\mathbf{y}_{\epsilon}\right)-u\left(\mathbf{y}_{0}\right) & =f(\epsilon)-f(0) \\
& =\int_{0}^{\epsilon} f^{\prime}(\eta) d \eta=\int_{0}^{\epsilon}\left[\frac{\partial u\left(\mathbf{y}_{\eta}\right)}{\partial \eta}\right] d \eta \\
& =\int_{0}^{\epsilon}\left[\sum_{s=1}^{T} u_{s}^{\prime}\left(\mathbf{y}_{\eta}\right) \cdot \frac{\partial y_{\eta}^{s}}{\partial \eta}\right] d \eta \\
& =\int_{0}^{\epsilon}\left[u_{t}^{\prime}\left(\mathbf{y}_{\eta}\right) \cdot 1+u_{t^{\prime}}^{\prime}\left(\mathbf{y}_{\eta}\right) \cdot-1\right] d \eta .
\end{aligned}
$$

(i) To prove the 'only if' part, assume $u \in \mathcal{V}$ and let $t<t^{\prime}$. Suppose contrary to what has to be proven that $u_{t}^{\prime}(\mathbf{z})<u_{t^{\prime}}^{\prime}(\mathbf{z})$ for some $\mathbf{z} \in \mathbb{R}^{T}$. Define $\mathbf{B}_{\theta}(\mathbf{z})$ as the $\theta$-neighborhood of the vector $\mathbf{z}$ for $\theta>0$. Because of continuity of the partial derivatives, there exists a $\theta>0$ such that $u_{t}^{\prime}\left(\mathbf{z}^{\prime}\right)<u_{t^{\prime}}^{\prime}\left(\mathbf{z}^{\prime}\right)$ for all $\mathbf{z}^{\prime} \in \mathbf{B}_{\theta}(\mathbf{z})$. Choose $\mathbf{y} \in \mathbb{R}^{T}$ and $\epsilon>0$ in such a way that $\mathbf{y}_{\eta} \in \mathbf{B}_{\theta}(\mathbf{z})$ for every $\eta \in[0, \epsilon]$, then by (1) it follows that $u\left(\mathbf{y}_{\epsilon}\right)-u\left(\mathbf{y}_{0}\right)<0$, which is a contradiction to $u \in \mathcal{V}$.

(ii) For the converse suppose that $u_{t}^{\prime}(\mathbf{x}) \geq u_{t^{\prime}}^{\prime}(\mathbf{x})$ for all $\mathbf{x} \in \mathbb{R}^{T}$. From (1) it then follows that $u\left(\mathbf{y}_{\epsilon}\right)-u\left(\mathbf{y}_{0}\right) \geq 0$, hence $u \in \mathcal{V}$.

A value $\psi: \mathcal{G}^{N} \rightarrow \mathbb{R}^{N}$ assigns to a TU-game $v \in \mathcal{G}^{N}$ the vector $\psi(v)$. The value $\psi$ is additive if for all $v, w \in \mathcal{G}^{N}$ it holds that $\psi(v+w)=\psi(v)+\psi(w)$. In case $\psi(v+w)=\psi(v)+\psi(w)$ holds for all $v, w \in \mathcal{G}^{N}$ where $w$ is additive, a weaker form of additivity of $\psi$ is considered. The value $\psi$ is restrictively additive if for all $v, w \in \mathcal{G}^{N}$ where $w$ is an additive game

\footnotetext{
${ }^{3}$ The notation $u_{t}^{\prime}(\mathbf{x})$ denotes the partial derivative of $u$ with respect to the $t$-th coordinate.
} 
generated by $\overline{\mathbf{w}}=(w(1), \ldots, w(n)) \in \mathbb{R}^{N}$ it holds that $\psi(v+w)=\psi(v)+\overline{\mathbf{w}}$. Hence, restricted additivity is a combination of the above mentioned weaker form of additivity and the condition that $\psi(w)=\overline{\mathbf{w}}$ for all additive games $w$ generated by $\overline{\mathbf{w}}=(w(1), \ldots, w(n))$. As a result restricted additivity is not necessarily weaker than additivity. For every $i \in N$, $\psi_{i}$ is concave if for all $v, w \in \mathcal{G}^{N}$ and $\lambda \in(0,1)$ it holds that $\psi_{i}(\lambda v+(1-\lambda) w) \geq$ $\lambda \psi_{i}(v)+(1-\lambda) \psi_{i}(w)$. For $i \in T \subseteq N, \psi_{i}$ is [strictly] decreasing in the worth of coalition $T$ if $\psi_{i}(v) \leq \psi_{i}\left(v^{\prime}\right)\left[\psi_{i}(v)<\psi_{i}\left(v^{\prime}\right)\right]$ for all $v, v^{\prime} \in \mathcal{G}^{N}$ with $v(T)>v^{\prime}(T)$ and $v(S)=v^{\prime}(S)$ for all $S \neq T$. The function $\psi_{i}$ is [strictly] increasing in the worth of coalition $T$ if $-\psi_{i}$ is [strictly] decreasing in the worth of coalition $T$.

The games $1_{T}, 2_{T} \in \mathcal{G}^{N}$ for $T \subset N$ are defined as follows. The game $1_{T}$ has worth $1_{T}(S)=1$ if $S=T$ and $1_{T}(S)=0$ otherwise and the game $2_{T}$ has worths $2_{T}(S)=1$ if $T \subseteq S$ and $2_{T}(S)=0$ otherwise. Note that the game $2_{T}$ is the usual unanimity game on $T$.

Clearly, the game $2_{i}{ }^{4}$ for $i \in N$ is an additive game generated by the vector $\overline{\mathbf{e}}_{i} \in \mathbb{R}^{N}$ which has a 1 for the element on place $i$ and zeros for all other elements. In case $\psi$ is restrictively additive and $\psi_{i}(z)=0$ for every $i \in N$ it follows that $\psi\left(\alpha 2_{i}\right)=\alpha \overline{\mathbf{e}}_{i}$ for every $i \in N$ and all $\alpha \in \mathbb{R}$. Thus, $\psi_{i}\left(\alpha 2_{i}\right)=\alpha$ and $\psi_{j}\left(\alpha 2_{i}\right)=0$ for all $i, j \in N, j \neq i$ and $\alpha \in \mathbb{R}$.

\section{Lemma 2}

(i) Let $\psi$ be additive and let $\psi_{i}(z)=0$ for all $i \in N$, then $\psi_{i}\left(\alpha 1_{i}\right)=\alpha \psi_{i}\left(1_{i}\right)$ for every $i \in N$ and all $\alpha \in \mathbb{Q}$.

(ii) If additionally, $\psi_{i}$ is continuous in $v(i)$ for all $i \in N$ and all $v \in \mathcal{G}^{N}$, then $\psi_{i}\left(\alpha 1_{i}\right)=$ $\alpha \psi_{i}\left(1_{i}\right)$ for every $i \in N$ and all $\alpha \in \mathbb{R}$.

Proof. (i) Let $i \in N$ and write $\psi_{i}\left(1_{i}\right)=r$. By additivity of $\psi$ and $\psi_{i}(z)=0$ we have $\psi_{i}\left(-1_{i}\right)+\psi_{i}\left(1_{i}\right)=\psi_{i}\left(-1_{i}+1_{i}\right)=\psi_{i}(z)=0$. Hence, $\psi_{i}\left(-1_{i}\right)=-\psi_{i}\left(1_{i}\right)=-r$. Вy additivity it then holds that $\psi_{i}\left(k 1_{i}\right)=k \psi_{i}\left(1_{i}\right)=k r$ for $k \in \mathbb{N}$ and in a similar way $\psi_{i}\left(-k 1_{i}\right)=k \psi_{i}\left(-1_{i}\right)=-k r$. For $\frac{p}{q} \in \mathbb{Q}, \psi_{i}\left(\frac{p}{q} 1_{i}\right)=\frac{1}{q} q \psi_{i}\left(\frac{p}{q} 1_{i}\right)=\frac{1}{q} \psi_{i}\left(\frac{p}{q} q 1_{i}\right)=\frac{1}{q} \psi_{i}\left(p 1_{i}\right)=$ $\frac{1}{q} p \psi_{i}\left(1_{i}\right)=\frac{p}{q} r$. Thus, $\psi_{i}\left(\alpha 1_{i}\right)=\alpha \psi_{i}\left(1_{i}\right)$ for all $\alpha \in \mathbb{Q}$.

(ii) let $\psi_{i}$ be continuous in $v(i)$ for all $i \in N$ and all $v \in \mathcal{G}^{N}$, which implies $\psi_{i}\left(\alpha 1_{i}\right)$ is continuous in $\alpha$. Combining this with part (i) it follows that $\psi_{i}\left(\alpha 1_{i}\right)=\alpha \psi_{i}\left(1_{i}\right)$ for all $\alpha \in \mathbb{R}$.

Instead of continuity in Lemma 2(ii), also monotonicity can be used.

Lemma 3 Let $\psi_{i}(z)=0$ for all $i \in N$. Let $\psi$ be additive and let $\psi_{i}$ be increasing in $v(i)$ for all $i \in N$ and all $v \in \mathcal{G}^{N}$. Then $\psi_{i}\left(\alpha 1_{i}\right)=\alpha \psi_{i}\left(1_{i}\right)$ for every $i \in N$ and all $\alpha \in \mathbb{R}$.

Proof. For $\alpha \in \mathbb{R}$, there exist $\frac{p}{q}, \frac{p^{\prime}}{q^{\prime}} \in \mathbb{Q}$ with $p, q, p^{\prime}, q^{\prime} \in \mathbb{Z}$ such that $\frac{p}{q}<\alpha<\frac{p^{\prime}}{q^{\prime}}$. Since $\psi_{i}$ is increasing in $v(i)$ for all $i \in N$ and $v \in \mathcal{G}^{N}$, it follows that $\psi_{i}\left(\alpha 1_{i}\right)$ is increasing in

\footnotetext{
${ }^{4}$ For simplicity a coalition is written without curly braces, e.g. $i j$ is written instead of $\{i, j\}$ for $i, j \in N$.
} 
$\alpha$. Since $\psi_{i}(z)=0$, as a result $\psi_{i}\left(1_{i}\right) \geq 0$. From Lemma $2(\mathrm{i})$ and because $\frac{p}{q}<\alpha<\frac{p^{\prime}}{q^{\prime}}$ it follows that $\psi_{i}\left(\frac{p}{q} 1_{i}\right)=\frac{p}{q} \psi_{i}\left(1_{i}\right) \leq \psi_{i}\left(\alpha 1_{i}\right) \leq \frac{p^{\prime}}{q^{\prime}} \psi_{i}\left(1_{i}\right)=\psi_{i}\left(\frac{p^{\prime}}{q^{\prime}} 1_{i}\right)$. The fractions $\frac{p}{q}, \frac{p^{\prime}}{q^{\prime}}$ can be chosen very close to $\alpha$, such that $\frac{p}{q}$ approaches $\alpha$ from below and $\frac{p^{\prime}}{q^{\prime}}$ approaches $\alpha$ from above. Thus, $\psi_{i}\left(\alpha 1_{i}\right)=\alpha \psi_{i}\left(1_{i}\right)$ for all $\alpha \in \mathbb{R}$.

In the next section a solution $\psi$ is applied to dynamic TU-games. This means that $\psi$ is applied to every TU-game within the dynamic TU-game separately. Individual intertemporal transfers are introduced which influence the exogenously determined dynamic TU-game. The strategies resulting from individual intertemporal transfers are modeled in a noncooperative game. Lemma 3 is used to find possible dominant strategies of this noncooperative game.

\section{Individual Intertemporal Transfers and Nash Equi- libria}

In this section it is assumed that players can make individual transfers between TU-games within a dynamic game, by which they can affect their intertemporal payoffs. If a player makes an individual transfer he transfers payoff over time: this payoff transfer affects certain worths in time which can have an affect on the intertemporal payoffs of the players. We assume that there is no discounting involved with these transfers, although the intertemporal utility function with which players evaluate their payoffs can be discounted utility. One way to think about this is that each player possesses a quantity of some good which does not loose its quality over time and can be stored. It can be transferred over time and still maintain its quality, however the utility which players receive from its consumption can change, in particular decrease, over time.

Two transfer systems are distinguished. Under the first transfer system, a player can transfer payoffs over time which only change the player's individual worths. In other words, by using a transfer a player can redistribute his individual worths over time. If payoffs are transferred according to the second transfer system, a player's transfer does not only change his individual worths, but also the worths of all coalitions that contain this player. In this latter system we assume that in a TU-game an individual transfer ceteris paribus changes all worths of coalitions which contain the individual by an amount equal to the individual transfer. This means that within a TU-game the change of a coalition's worth equals the sum of the changes of the relevant individual worths. In order to ensure that the worths of all coalitions remain nonnegative under the second transfer system, only dynamic games with weakly superadditive TU-games are considered. Although it is possible to impose a less stringent condition to guarantee nonnegativity of all TU-games, we use weak superadditivity to ensure that the strategy set of a player under the first transfer system equals the strategy set of the player under the second transfer system. By imposing weak superadditivity it follows that the worth of a coalition consists of the individual worths of the players which are contained in the coalition and a nonnegative worth generated by a 
nonnegative synergy resulting from the fact that players work together. The interpretation that the worth of a coalition thus can be seen as a transferable part namely the individual worths of the relevant players and a nontransferable part which is the worth resulting from a nonnegative synergy, supports the second transfer system.

Definition 3: With each $\Gamma=(\mathbf{v}, \mathbf{u})$ and value $\psi$ we associate for $k \in\{1,2\}$ the noncooperative game $G_{k}(\Gamma, \psi)=\left(\mathcal{S}_{1}, \ldots, \mathcal{S}_{n} ; \tilde{u}_{1}, \ldots, \tilde{u}_{n}\right)$ with the extra condition that if $k=2$ then $v^{t} \in \mathcal{G}^{N}$ is weakly superadditive for all $t \in\{1, \ldots, T\}$. For every $i \in N$,

(i) strategy set $\mathcal{S}_{i}=\left\{\alpha_{i} \in \mathbb{R}^{T} \mid v^{t}(i)+\alpha_{i}^{t} \geq 0\right.$ for all $t \in\{1, \ldots, T\}$ and $\left.\sum_{t=1}^{T} \alpha_{i}^{t}=0\right\}$.

(ii) utility function $\tilde{u}_{i}: \mathcal{S}_{N}{ }^{5} \rightarrow \mathbb{R}$ where

- for $k=1$ it holds that $\tilde{u}_{i}\left(\alpha_{1}, \ldots, \alpha_{n}\right)=u_{i}\left(\psi_{i}\left(v^{1}+\sum_{j=1}^{n} \alpha_{j}^{1} 1_{j}\right), \ldots, \psi_{i}\left(v^{T}+\sum_{j=1}^{n} \alpha_{j}^{T} 1_{j}\right)\right)$.

- for $k=2$ it holds that $\tilde{u}_{i}\left(\alpha_{1}, \ldots, \alpha_{n}\right)=u_{i}\left(\psi_{i}\left(v^{1}+\sum_{j=1}^{n} \alpha_{j}^{1} 2_{j}\right), \ldots, \psi_{i}\left(v^{T}+\sum_{j=1}^{n} \alpha_{j}^{T} 2_{j}\right)\right)$.

Nash equilibria of the noncooperative game $G_{k}(\Gamma, \psi)$ for $k \in\{1,2\}$ are the main interest of this paper. A Nash equilibrium is a strategy profile $\left(\alpha_{1}^{*}, \ldots, \alpha_{n}^{*}\right) \in \mathcal{S}_{N}$ such that for each $i \in N$ it holds that $\tilde{u}_{i}\left(\alpha_{i}, \alpha_{N \backslash i}^{*}\right) \leq \tilde{u}_{i}\left(\alpha_{i}^{*}, \alpha_{N \backslash i}^{*}\right)$ for all $\alpha_{i} \in \mathcal{S}_{i}$. However, a Nash equilibrium does not have to exist as Example 4 later shows.

First, attention is given to weakly and strictly dominant strategies. A strategy $\alpha_{i}^{*} \in \mathcal{S}_{i}$ of player $i$ is weakly dominant if $\tilde{u}_{i}\left(\alpha_{i}^{*}, \alpha_{N \backslash i}\right) \geq \tilde{u}_{i}\left(\alpha_{i}, \alpha_{N \backslash i}\right)$ for all $\alpha_{i} \in \mathcal{S}_{i}$ and all $\alpha_{N \backslash i} \in \mathcal{S}_{N \backslash i}$. If $\tilde{u}_{i}\left(\alpha_{i}^{*}, \alpha_{N \backslash i}\right)>\tilde{u}_{i}\left(\alpha_{i}, \alpha_{N \backslash i}\right)$ for all $\alpha_{i} \in \mathcal{S}_{i}$ and all $\alpha_{N \backslash i} \in \mathcal{S}_{N \backslash i}$, then $\alpha_{i}^{*} \in \mathcal{S}_{i}$ is player $i$ 's strictly dominant strategy. Observe that a combination of weakly dominant strategies is a Nash equilibrium. If these strategies are strictly dominant then this Nash equilibrium is unique.

Theorem 1 Let $\Gamma=(\mathbf{v}, \mathbf{u})$ be a dynamic game and let $\psi$ be additive. Let $i \in N$ and let $\psi_{i}(z)=0$.

(i) If $u_{i} \in \mathcal{V}$ and if $\psi_{i}$ is increasing in $v(i)$ for all $v \in \mathcal{G}^{N}$, then $\left(\sum_{t=2}^{T} v^{t}(i),-v^{2}(i), \ldots,-v^{T}(i)\right)$ is a weakly dominant strategy for player $i$ in $G_{1}(\Gamma, \psi)$.

(ii) If $u_{i} \in \overline{\mathcal{V}}$ and if $\psi_{i}$ is strictly increasing in $v(i)$ for all $v \in \mathcal{G}^{N}$, then $\left(\sum_{t=2}^{T} v^{t}(i),-v^{2}(i), \ldots,-v^{T}(i)\right)$ is player $i$ 's strictly dominant strategy in $G_{1}(\Gamma, \psi)$.

Proof. (i) Let $\psi_{i}$ be increasing in $v(i)$ for all $v \in \mathcal{G}^{N}$. To determine player $i$ 's dominant strategies, player $i$ is fixed and the strategies of all other players $N \backslash i$ are considered to be given. Without loss of generality it is assumed that the game $v^{t} \in \mathcal{G}^{N}$ for every $t$ includes the transfers resulting from all strategies of players $N \backslash i$. By additivity of $\psi$ we can assume without loss of generality that $v^{t}=z$ for all $t$. Because $\psi_{i}$ is increasing in $v(i)$

\footnotetext{
${ }^{5} \mathcal{S}_{T}=\times_{i \in T} \mathcal{S}_{i}$ for all $T \subseteq N$
} 
for all $v \in \mathcal{G}^{N}$ and $\psi_{i}(z)=0$, it follows that $\psi_{i}\left(1_{i}\right) \geq 0$. From Lemma 3 it follows that $u_{i}\left(\psi_{i}\left(v^{1}+\alpha_{i}^{1} 1_{i}\right), \ldots, \psi_{i}\left(v^{T}+\alpha_{i}^{T} 1_{i}\right)\right)=u_{i}\left(\alpha_{i}^{1} \psi_{i}\left(1_{i}\right), \ldots, \alpha_{i}^{T} \psi_{i}\left(1_{i}\right)\right)$ for all $\alpha_{i} \in \mathcal{S}_{i}$.

Take two arbitrary strategies $\beta, \gamma \in \mathcal{S}_{i}$ with $\beta^{t}-\gamma^{t} \leq 0$ for all $t \neq 1$. Then it follows that

$$
\begin{aligned}
u_{i}\left(\beta^{1} \psi_{i}\left(1_{i}\right), \ldots, \beta^{T} \psi_{i}\left(1_{i}\right)\right) & =u_{i}\left(\psi_{i}\left(1_{i}\right)\left(\beta^{1}, \ldots, \beta^{T}\right)\right) \\
& \geq u_{i}\left(\psi_{i}\left(1_{i}\right)\left(\beta^{1}, \ldots, \beta^{T-1}+\left(\beta^{T}-\gamma^{T}\right), \gamma^{T}\right)\right) \\
& \vdots \\
& \geq u_{i}\left(\psi_{i}\left(1_{i}\right)\left(\beta^{1}+\sum_{t=2}^{T}\left(\beta^{t}-\gamma^{t}\right), \ldots, \gamma^{T}\right)\right) \\
& =u_{i}\left(\psi_{i}\left(1_{i}\right)\left(\gamma^{1}, \ldots, \gamma^{T}\right)\right) .
\end{aligned}
$$

The first equality follows from scalar multiplication. The first inequality follows from $u_{i} \in \mathcal{V}$ and because $\left(\beta^{T}-\gamma^{T}\right) \psi_{i}\left(1_{i}\right) \leq 0$. The dots represent the second till the penultimate inequality. The second inequality follows from $u_{i} \in \mathcal{V}$ and because $\left(\beta^{T-1}-\right.$ $\left.\gamma^{T-1}\right) \psi_{i}\left(1_{i}\right) \leq 0$, etc. The equality at the end follows from $\sum_{t=1}^{T} \beta^{t}=\sum_{t=1}^{T} \gamma^{t}=0$. Because $\beta, \gamma \in \mathcal{S}_{i}$ it holds that $\beta^{t}, \gamma^{t} \geq-v^{t}(i)$ for all $t$. Choose $\left(\beta^{1}, \beta^{2}, \ldots, \beta^{T}\right)=$ $\left(\sum_{t=2}^{T} v^{t}(i),-v^{2}(i), \ldots,-v^{T}(i)\right)$, then (2) holds for all $\gamma \in \mathcal{S}_{i}$ because $\beta^{t}-\gamma^{t} \leq 0$ for all $t \neq 1$. As a result $\left(\sum_{t=2}^{T} v^{t}(i),-v^{2}(i), \ldots,-v^{T}(i)\right)$ is a weakly dominant strategy for player $i$ in $G_{1}(\Gamma, \psi)$.

(ii) Let $\psi_{i}$ be strictly increasing in $v(i)$ for all $v \in \mathcal{G}^{N}$, then $\psi_{i}\left(1_{i}\right)>0$ since $\psi_{i}(z)=0$. The remainder of the proof of part (ii) follows the proof of part (i), however we take two arbitrary strategies $\beta, \gamma \in \mathcal{S}_{i}$ with $\beta^{t}-\gamma^{t} \leq 0$ for all $t \neq 1$ and $\beta^{t}-\gamma^{t}<0$ for at least one $t \neq 1$. Because $u_{i} \in \overline{\mathcal{V}}$ it then follows that at least one of the inequality signs $(\geq)$ in (2) should be replaced by a strict inequality sign $(>)$. As a result $\left(\sum_{t=2}^{T} v^{t}(i),-v^{2}(i), \ldots,-v^{T}(i)\right)$ is player $i$ 's strictly dominant strategy in $G_{1}(\Gamma, \psi)$.

Theorem 1 shows that the strategy to transfer as much payoff as possible to the first time period is a weakly dominant strategy for player $i$. The next theorem shows in which case the condition $\psi_{i}$ is increasing in the individual worth of player $i$ is a necessary condition.

Theorem 2 Let $i \in N$, let $u_{i}: \mathbb{R}^{T} \rightarrow \mathbb{R}$ and let $u_{i} \in \mathcal{V}$. Let $\psi$ be additive, let $\psi_{i}(z)=0$ and let $\psi_{i}$ be continuous in $v(i)$ for all $v \in \mathcal{G}^{N}$. Then, if $\left(\sum_{t=2}^{T} v^{t}(i),-v^{2}(i), \ldots,-v^{T}(i)\right)$ is a weakly dominant strategy of player $i$ for all $\Gamma=(\mathbf{v}, \mathbf{u})$ in $G_{1}(\Gamma, \psi)$, then $\psi_{i}$ is increasing in $v(i)$ for all $v \in \mathcal{G}^{N}$.

Proof. Fix $i \in N$ and let $\left(\sum_{t=2}^{T} v^{t}(i),-v^{2}(i), \ldots,-v^{T}(i)\right)$ be a weakly dominant strategy of player $i$ in $G_{1}(\Gamma, \psi)$ for all $\Gamma=(\mathbf{v}, \mathbf{u})$. Suppose that there exist $v, v^{\prime} \in \mathcal{G}^{N}$ with $v(i)>v^{\prime}(i), v(S)=v^{\prime}(S)$ for all $S \neq\{i\}$ and $\psi_{i}(v)<\psi_{i}\left(v^{\prime}\right)$. By additivity of $\psi$ and since $\psi$ is continuous in the individual worth of player $i$ it follows that $\left(v(i)-v^{\prime}(i)\right) \psi_{i}\left(1_{i}\right)<0$, hence $\psi_{i}\left(1_{i}\right)<0$. Therefore it follows for every $w, w^{\prime} \in \mathcal{G}^{N}$ with $w(i)>w^{\prime}(i)$ and $w(S)=$ $w^{\prime}(S)=v(S)$ for every $S \neq\{i\}$ that $\psi_{i}(w)=w(i) \psi_{i}\left(1_{i}\right)<w^{\prime}(i) \psi_{i}\left(1_{i}\right)=\psi_{i}\left(w^{\prime}\right)$. Take $w, w^{\prime}$ with $w(i)=1, w^{\prime}(i)=0$ and $w(S)=w^{\prime}(S)=v(S)$ for every $S \neq\{i\}$. Consider the 
sequence of stage games $\left(w, w^{\prime}, z, \ldots, z\right) \in\left(\mathcal{G}^{N}\right)^{T}$. In this case $\left(w^{\prime}(i),-w^{\prime}(i), 0, \ldots, 0\right)=$ $(0,0, \ldots, 0) \in \mathcal{S}_{i}$ is a weakly dominant strategy of player $i$. It follows that

$$
\begin{aligned}
& u_{i}\left(\psi_{i}(w), \psi_{i}\left(w^{\prime}\right), \psi_{i}(z), \ldots, \psi_{i}(z)\right) \\
\geq & u_{i}\left(\psi_{i}\left(w+\left(w^{\prime}(i)-w(i)\right) 1_{i}\right), \psi_{i}\left(w^{\prime}-\left(w^{\prime}(i)-w(i)\right) 1_{i}\right), \ldots, \psi_{i}(z)\right) \\
= & u_{i}\left(\psi_{i}\left(w^{\prime}\right), \psi_{i}(w), \psi_{i}(z), \ldots, \psi_{i}(z)\right) .
\end{aligned}
$$

Inequality (3) is true only if $\left(w^{\prime}(i)-w(i)\right) \psi_{i}\left(1_{i}\right) \leq 0$, which is a contradiction since $w^{\prime}(i)-w(i)=-1$ and $\psi_{i}\left(1_{i}\right)<0$. Therefore $\psi_{i}(w) \geq \psi_{i}\left(w^{\prime}\right)$. Thus, $\psi_{i}$ is increasing in $w(i)$ for all $w \in \mathcal{G}^{N}$.

In the next theorem we consider noncooperative game $G_{2}(\Gamma, \psi)$ in which an individual intertemporal transfer influences all worths of coalitions which contain the individual. We assume that within a TU-game the change of a coalition's worth equals the sum of the amounts of the relevant individual transfers. This means that in case the individual transfers at time $t$ are denoted by $\alpha_{i}^{t} \in \mathcal{S}_{i}$ for all $i \in N$, then all worths of coalitions $S \subseteq N$ change with $\sum_{i \in S} \alpha_{i}^{t}$ at $t$. Hence, the worth of coalition $S \subseteq N$ at $t$ after transfers are applied equals $v^{t}(S)+\sum_{i \in N} \alpha_{i}^{t} 2_{i}(S)$, where $v^{t}(S)$ is the worth before payoffs are transferred and $\sum_{i \in N} \alpha_{i}^{t} 2_{i}$ is an additive game generated by the vector $\left(\alpha_{1}^{t}, \ldots, \alpha_{n}^{t}\right) \in \mathbb{R}^{N}$. Because an additive game is generated by the individual intertemporal transfers of the players, a restrictively additive solution can be used in the next theorem to obtain a weakly dominant strategy for player $i$.

Theorem 3 Let $\Gamma=(\mathbf{v}, \mathbf{u})$ be a dynamic game, let $v^{t} \in \mathcal{G}^{N}$ be weakly superadditive for all $t \in\{1, \ldots, T\}$ and let $\psi$ be restrictively additive. Let $i \in N$ and let $\psi_{i}(z)=0$.

(i) If $u_{i} \in \mathcal{V}$, then $\left(\sum_{t=2}^{T} v^{t}(i),-v^{2}(i), \ldots,-v^{T}(i)\right)$ is a weakly dominant strategy for player $i$ in $G_{2}(\Gamma, \psi)$.

(ii) If $u_{i} \in \overline{\mathcal{V}}$, then $\left(\sum_{t=2}^{T} v^{t}(i),-v^{2}(i), \ldots,-v^{T}(i)\right)$ is player $i$ 's strictly dominant strategy in $G_{2}(\Gamma, \psi)$.

Proof. (i) Fix player $i$. For all $\alpha \in \mathcal{S}_{N}$ it holds that $\psi_{i}\left(v^{t}+\sum_{j=1}^{N} \alpha_{j}^{t} 2_{j}\right)=\psi_{i}\left(v^{t}\right)+\alpha_{i}^{t}$ for all $j \in N$ and all $t$, because $\psi$ is restrictively additive and $\psi_{i}(z)=0$. Take two arbitrary strategies $\beta, \gamma \in \mathcal{S}_{i}$ with $\beta^{t}-\gamma^{t} \leq 0$ for $t \neq 1$. Then it follows that

$$
\begin{aligned}
& u_{i}\left(\psi_{i}\left(v^{1}\right)+\beta^{1}, \ldots, \psi_{i}\left(v^{T}\right)+\beta^{T}\right) \\
\geq & u_{i}\left(\psi_{i}\left(v^{1}\right)+\beta^{1}, \ldots, \psi_{i}\left(v^{T-1}\right)+\beta^{T-1}+\beta^{T}-\gamma^{T}, \psi_{i}\left(v^{T}\right)+\gamma^{T}\right) \\
\vdots & \\
\geq & u_{i}\left(\psi_{i}\left(v^{1}\right)+\beta^{1}+\sum_{t=2}^{T}\left(\beta^{t}-\gamma^{t}\right), \psi_{i}\left(v^{2}\right)+\gamma^{2}, \ldots, \psi_{i}\left(v^{T}\right)+\gamma^{T}\right) \\
= & u_{i}\left(\psi_{i}\left(v^{1}\right)+\gamma^{1}, \ldots, \psi_{i}\left(v^{T}\right)+\gamma^{T}\right) .
\end{aligned}
$$


The first inequality follows from $u_{i} \in \mathcal{V}$ and since $\beta^{T}-\gamma^{T} \leq 0$. The vertical dots represent the second till the penultimate inequality. The second inequality follows from $u_{i} \in \mathcal{V}$ and because $\beta^{T-1}-\gamma^{T-1} \leq 0$, etc. The last inequality follows from $u_{i} \in \mathcal{V}$ and $\beta^{2}-\gamma^{2} \leq 0$. Finally, the equality follows because $\sum_{t=1}^{T} \beta^{t}=\sum_{t=1}^{T} \gamma^{t}=0$. Since $\beta, \gamma \in \mathcal{S}_{i}$ and $v^{t} \in \mathcal{G}^{N}$ is weakly superadditive for all $t$, it follows that $\beta^{t}, \gamma^{t} \geq-v^{t}(i)$ for all $i \in N$ and all $t$. Hence, if $\left(\beta^{1}, \beta^{2}, \ldots, \beta^{T}\right)$ is chosen equal to $\left(\sum_{t=2}^{T} v^{t}(i),-v^{2}(i), \ldots,-v^{T}(i)\right)$, then for all $\gamma \in \mathcal{S}_{i}$ it follows that (4) holds, since $\beta^{t}-\gamma^{t} \leq 0$ for $t \neq 1$. From (4) it follows that $\left(\sum_{t=2}^{T} v^{t}(i),-v^{2}(i), \ldots,-v^{T}(i)\right)$ is a weakly dominant strategy for player $i$ in $G_{2}(\Gamma, \psi)$.

(ii) The proof of part (ii) follows the line of proof of part (i), however take $\beta^{t}-\gamma^{t} \leq 0$ for all $t \neq 1$ and $\beta^{t}-\gamma^{t}<0$ for at least one $t \neq 1$. Because $u_{i} \in \overline{\mathcal{V}}$ it then follows that at least one of the inequality signs $(\geq)$ in $(4)$ should be replaced by a strict inequality sign $(>)$. In a similar way as in part (i) we then find that $\left(\sum_{t=2}^{T} v^{t}(i),-v^{2}(i), \ldots,-v^{T}(i)\right)$ is player $i$ 's strictly dominant strategy in $G_{2}(\Gamma, \psi)$.

If the assumption $v^{t}(S)-\max _{t^{\prime} \in\{1, \ldots, T\}} \sum_{j \in S} v^{t^{\prime}}(j) \geq 0$ for all $S \subseteq N$ is imposed on $v^{t} \in \mathcal{G}^{N}$ for all $t$ in Theorem 3 instead of the stricter condition of weakly superadditivity on $v^{t} \in \mathcal{G}^{N}$ for all $t$, then it does not have to be true that $\left(\sum_{t=2}^{T} v^{t}(i),-v^{2}(i), \ldots,-v^{T}(i)\right)$ is contained in player $i$ 's newly obtained strategy set. However, in case $u_{i} \in \mathcal{V}$ it follows that a strategy, contained in this newly obtained strategy set of player $i$, where as much as possible is transferred to the first time period is a weakly dominant strategy for player $i$. In case $u_{i} \in \overline{\mathcal{V}}$ transferring as much as possible to the first time period is player $i$ 's strictly dominant strategy.

Obviously, if the conditions in Theorems 1 and 3 apply to all players, then there is at least one Nash equilibrium in dominant strategies. In this equilibrium all players transfer as much payoff as possible to the first time period. However, players do not have to value the same time period as most important. If for some (or all) players the first time period is not as important as another time period, then a Nash equilibrium in dominant strategies is obtained in which every player transfers as much payoff as possible to his most important time period.

In Theorem 3 a restrictively additive solution is considered. The nucleolus and prenucleolus are restrictively additive. Specifically, they are covariant (Peleg and Sudhölter, 2003). The nucleolus is a one-point payoff distribution which is defined for games in $\mathcal{G}^{N}$ with a nonempty imputation set. The imputation set contains all payoffs which are efficient and individually rational for all players. If the core of a game is nonempty then the nucleolus is in the core. In case the core is nonempty, the nucleolus and prenucleolus coincide. The prenucleolus is also defined if the imputation set is empty. As a matter of fact, it always exists.

Both solutions are defined as the solution of a minimization problem, where excesses of all nonempty coalitions of a game are lexicographically minimized. The excess of a coalition $S$ in game $v \in \mathcal{G}^{N}$ given a payoff vector $\mathbf{x}=(x(1), \ldots, x(n)) \in \mathbb{R}^{N}$ is defined as $v(S)$ $\sum_{i \in S} x(i)$ for all $v \in \mathcal{G}^{N}$. The nucleolus as well as the prenucleolus satisfy the efficiency property, i.e. $\sum_{i \in N} x(i)=v(N)$. Additionally, the nucleolus satisfies the property of 
individual rationality for all players which means that $x(i) \geq v(i)$ for all $i \in N{ }^{6}$

If the (pre)nucleolus is applied to the dynamic game $\Gamma=(\mathbf{v}, \mathbf{u})$ where $u_{i} \in \mathcal{V}$ for player $i \in N$, then transferring as much payoff as possible to the first time period is a weakly dominant strategy of player $i$ in case payoffs are transferred according to the second transfer system. However, as the following example illustrates, this implication does not have to hold in case the first transfer system is used. In particular it is shown that transferring as much as possible to $t=1$ is not at all beneficial for player $i$.

Example 1. Consider the dynamic TU-game $\Gamma=\left(v^{1}, v^{2} ; u_{1}, u_{2}, u_{3}\right)$ and transfer system $k=1$. The game $v^{1}$ is defined as follows: $v^{1}(1)=0 ; v^{1}(2)=2 ; v^{1}(3)=3 ; v^{1}(12)=4$; $v^{1}(13)=v^{1}(23)=5$ and $v^{1}(N)=7$, and the game $v^{2}$ has $v^{2}(1)=1 ; v^{2}(2)=v^{2}(3)=0$; $v^{2}(12)=v^{2}(13)=v^{2}(23)=2$ and $v^{2}(N)=4$. The functions $u_{1}, u_{2}$ and $u_{3}$ are respectively player 1, player 2 and player 3's intertemporal utility functions. Furthermore $\nu\left(v^{1}\right)=$ $(2,2,3)$ and $\nu\left(v^{2}\right)=\left(\frac{3}{2}, \frac{5}{4}, \frac{5}{4}\right)$. Suppose that player 1 transfers $\alpha=(1,-1)$, which means that he transfers the maximum amount of payoff possible to his first time period. Then the games $v^{1}+\alpha^{1} 1_{1}$ and $v^{2}+\alpha^{2} 1_{1}$ are obtained and accordingly $\nu\left(v^{1}+1 \cdot 1_{1}\right)=(2,2,3)$ and $\nu\left(v^{2}-1 \cdot 1_{1}\right)=\left(\frac{4}{3}, \frac{4}{3}, \frac{4}{3}\right)$. Thus, player 1 receives payoff 2 in the first period and payoff $\frac{3}{2}$ in the second, before any transfer is made. After he makes the transfer $\alpha=(1,-1)$, he receives a payoff equal to 2 in the first period and only a payoff of $\frac{4}{3}$ in the second period. Under any reasonable utility function, transferring $(1,-1)$ is clearly not beneficial for player 1.

Since the cores of the TU-games $v^{1}$ and $v^{2}$ in example 1 are nonempty before as well as after player 1 has made a transfer, the prenucleolus and nucleolus coincide. Therefore this example also applies to the prenucleolus. In example 2, the first transfer system is applied to the dynamic game $\Gamma=(\mathbf{v}, \mathbf{u})$ over which the nucleolus is calculated to show that an extreme strategy profile (i.e a strategy profile where all payoff is transferred to either the first time period or the last time period) does not have to be a Nash equilibrium. As in example 1 the cores for the TU-games $v^{1}$ and $v^{2}$ before as well as after payoffs are transferred are nonempty and therefore the nucleolus and prenucleolus coincide.

Example 2. The dynamic TU-game $\Gamma=\left(v^{1}, v^{2} ; u_{1}, u_{2}, u_{3}\right)$ is considered over which the first transfer system is applied and where $u_{1}, u_{2}$ and $u_{3}$ are respectively player 1 , player 2 and player 3's intertemporal utility functions. The function $u_{1}$ is a constant discounted utility function with discount factor $0 \leq \delta \leq 1$. The game $v^{1}$ is described by $v^{1}(1)=v^{1}(2)=v^{1}(3)=0$ and $v^{1}(12)=v^{1}(13)=v^{1}(23)=\frac{2}{3}$ and $v^{1}(N)=3$. The game $v^{2}$ is defined as $v^{2}(1)=2$ and $v^{2}(S)=v^{1}(S)$ for all $S \neq\{1\}$. Clearly only player 1 can make transfers between the two time periods, which are denoted by $\alpha \in \mathbb{R}^{2}$ where $\alpha^{1}+\alpha^{2}=0$ and $\alpha^{2} \geq-2$.

\footnotetext{
${ }^{6}$ See Peleg and Sudhölter (2003) for more information about the nucleolus and prenucleolus.
} 


\begin{tabular}{lrr}
\hline$\alpha^{1}$ & $\nu\left(v^{1}+\alpha^{1} 1_{1}\right)$ & $\nu\left(v^{2}+\alpha^{2} 1_{1}\right)$ \\
\hline 0 & $(1,1,1)$ & $\left(2 \frac{1}{6}, \frac{5}{12}, \frac{5}{12}\right)$ \\
1 & $\left(1 \frac{2}{3}, \frac{2}{3}, \frac{2}{3}\right)$ & $\left(1 \frac{2}{3}, \frac{2}{3}, \frac{2}{3}\right)$ \\
2 & $\left(2 \frac{1}{6}, \frac{5}{12}, \frac{5}{12}\right)$ & $(1,1,1)$ \\
\hline
\end{tabular}

Table 1: Computation of nucleoli for different transfers.

Utility function $u_{1}\left(\nu\left(v^{1}+\alpha^{1} 1_{1}\right), \nu\left(v^{2}+\alpha^{2} 1_{1}\right)\right)=\nu\left(v^{1}+\alpha^{1} 1_{1}\right)+\delta \nu\left(v^{2}+\alpha^{2} 1_{1}\right)$ is contained in the class $\mathcal{V}$ for $\delta \leq 1$. If $\frac{3}{4}<\delta \leq 1$, then none of the two extreme transfers $\alpha^{1}=0$ and $\alpha^{1}=2$ give player 1 the highest possible utility.

Debreu (1952) proves the existence of a Nash equilibrium in a noncooperative game with finitely many players, whose strategy sets are convex and compact subsets of a Euclidian space and whose utility functions are continuous and quasi-concave. To apply Debreu's theorem to the noncooperative game $G_{k}(\Gamma, \psi)$ for $k \in\{1,2\}$ extra conditions are necessary in order to prove existence.

The value $\psi$ is transfer- 1 -concave if $\psi_{i}\left(\lambda(v+w)+(1-\lambda)\left(v+w^{\prime}\right)\right) \geq \lambda \psi_{i}(v+w)+(1-$ $\lambda) \psi_{i}\left(v+w^{\prime}\right)$ for all $i \in N$ and all $v, w, w^{\prime} \in \mathcal{G}^{N}$ where for $(w(1), \ldots, w(n)) \in \mathbb{R}^{N}$ and $\left(w^{\prime}(1), \ldots, w^{\prime}(n)\right) \in \mathbb{R}^{N}$ we have $w=\sum_{i=1}^{n} w(i) \mathbf{1}_{i}$ and $w^{\prime}=\sum_{i=1}^{n} w^{\prime}(i) \mathbf{1}_{i}$. The value $\psi$ is transfer-2-concave if $\psi_{i}\left(\lambda(v+w)+(1-\lambda)\left(v+w^{\prime}\right)\right) \geq \lambda \psi_{i}(v+w)+(1-\lambda) \psi_{i}\left(v+w^{\prime}\right)$ for all $i \in N$ and all $v, w, w^{\prime} \in \mathcal{G}^{N}$ where $w, w^{\prime}$ are additive, generated by respectively $(w(1), \ldots, w(n)) \in \mathbb{R}^{N}$ and $\left(w^{\prime}(1), \ldots, w^{\prime}(n)\right) \in \mathbb{R}^{N}$ which means that $w=\sum_{i=1}^{n} w(i) 2_{i}$ and $w^{\prime}=\sum_{i=1}^{n} w^{\prime}(i) 2_{i}$.

Lemma 4 Let $i \in N$ and let $k \in\{1,2\}$. If $\psi$ is transfer- $k$-concave and $u_{i}$ is an increasing and quasi-concave function, then $\tilde{u}_{i}$ is quasi-concave.

Proof. (i) Let $\psi$ be transfer-1-concave. This means that for all $\beta, \gamma \in \mathcal{S}_{N}$ and $\lambda \in(0,1)$ it holds that $\psi_{i}\left(\lambda\left(v^{t}+\sum_{j=1}^{n} \beta_{j}^{t} 1_{j}\right)+(1-\lambda)\left(v^{t}+\sum_{j=1}^{n} \gamma_{j}^{t} 1_{j}\right)\right) \geq \lambda \psi_{i}\left(v^{t}+\sum_{j=1}^{n} \beta_{j}^{t} 1_{j}\right)+(1-$ त) $\psi_{i}\left(v^{t}+\sum_{j=1}^{n} \gamma_{j}^{t} 1_{j}\right)$ for all $t$ and all $i \in N$. Then,

$$
\begin{aligned}
\tilde{u}_{i}(\lambda \beta+(1-\lambda) \gamma)= & u_{i}\left(\psi_{i}\left(v^{1}+\sum_{j=1}^{n}\left[\lambda \beta_{j}^{1}+(1-\lambda) \gamma_{j}^{1}\right] 1_{j}\right), \ldots, \psi_{i}\left(v^{T}+\sum_{j=1}^{n}\left[\lambda \beta_{j}^{T}+(1-\lambda) \gamma_{j}^{T}\right] 1_{j}\right)\right) \\
= & u_{i}\left(\psi_{i}\left(\lambda\left[v^{1}+\sum_{j=1}^{n} \beta_{j}^{1} 1_{j}\right]+(1-\lambda)\left[v^{1}+\sum_{j=1}^{n} \gamma_{j}^{1} 1_{j}\right]\right), \ldots\right. \\
& \left.\ldots, \psi_{i}\left(\lambda\left[v^{T}+\sum_{j=1}^{n} \beta_{j}^{T} 1_{j}\right]+(1-\lambda)\left[v^{T}+\sum_{j=1}^{n} \gamma_{j}^{T} 1_{j}\right]\right)\right) \\
\geq & u_{i}\left(\lambda \psi_{i}\left(v^{1}+\sum_{j=1}^{n} \beta_{j}^{1} 1_{j}\right)+(1-\lambda) \psi_{i}\left(v^{1}+\sum_{j=1}^{n} \gamma_{j}^{1} 1_{j}\right), \ldots\right. \\
& \left.\ldots, \lambda \psi_{i}\left(v^{T}+\sum_{j=1}^{n} \beta_{j}^{T} 1_{j}\right)+(1-\lambda) \psi_{i}\left(v^{T}+\sum_{j=1}^{n} \gamma_{j}^{T} 1_{j}\right)\right)
\end{aligned}
$$




$$
\begin{aligned}
\geq & \min \left\{u_{i}\left(\psi_{i}\left(v^{1}+\sum_{j=1}^{n} \beta_{j}^{1} 1_{j}\right), \ldots, \psi_{i}\left(v^{T}+\sum_{j=1}^{n} \beta_{j}^{T} 1_{j}\right)\right),\right. \\
& \left.u_{i}\left(\psi_{i}\left(v^{1}+\sum_{j=1}^{n} \gamma_{j}^{1} 1_{j}\right), \ldots, \psi_{i}\left(v^{T}+\sum_{j=1}^{n} \gamma_{j}^{T} 1_{j}\right)\right)\right\} \\
= & \min \left\{\tilde{u}_{i}(\beta), \tilde{u}_{i}(\gamma)\right\} .
\end{aligned}
$$

The first equality follows from the definition of $\tilde{u}_{i}$. The first inequality follows from transfer1-concavity of $\psi$ and because $u_{i}$ is an increasing function. The second inequality follows from quasi-concavity of $u_{i}$. Finally, the last equality follows from the definition of $\tilde{u}_{i}$.

(ii) Let $\psi$ be transfer-2-concave. Then $1_{j}$ in part (i) is replaced by $2_{j}$. In an analogous way as part (i) it follows that $\tilde{u}_{i}$ is quasi-concave.

Lemma 4 is used in Theorem 4 below to prove existence of a Nash equilibrium.

Theorem 4 Let $\Gamma=(\mathbf{v}, \mathbf{u})$ be a dynamic game. If $u_{i}$ is a continuous, increasing and quasi-concave function for all $i \in N$, if $\psi$ is continuous and

(i) if $\psi$ is transfer-1-concave, then a Nash equilibrium exists in $G_{1}(\Gamma, \psi)$.

(ii) if $\psi$ is transfer-2-concave and $v^{t} \in \mathcal{G}^{N}$ is weakly superadditive for all $t \in\{1, \ldots, T\}$, then a Nash equilibrium exists in $G_{2}(\Gamma, \psi)$.

Proof. (i) Let $\psi$ be continuous and transfer-1-concave and let $u_{i}$ be a continuous, increasing and quasi-concave function for all $i \in N$. From continuity of $\psi$ and continuity of $u_{i}$ it follows that $\tilde{u}_{i}$ is a continuous function. Since the set $\mathcal{S}_{i}$ is compact for all $i \in N$ it follows that $\mathcal{S}_{N}$ is compact. A convex combination of strategies in $\mathcal{S}_{N}$ is contained in $\mathcal{S}_{N}$ and therefore $\mathcal{S}_{N}$ is convex. From Debreu (1952) and Lemma 4 it follows that a Nash Equilibrium exists in $G_{1}(\Gamma, \psi)$.

(ii) The proof op part (ii) follows the line of proof of part (i). However, the assumption that $\psi$ is transfer-1-concave is replaced by the assumption that $\psi$ is transfer-2-concave. Furthermore let $v^{t} \in \mathcal{G}^{N}$ be weakly superadditive for all $t \in\{1, \ldots, T\}$. In an analogous way as (i) it then follows that a Nash Equilibrium exists in $G_{2}(\Gamma, \psi)$.

One of the conditions in Lemma 4 and Theorem 4 is transfer- $k$-concavity of the solution $\psi$. For $k \in\{1,2\}, \psi$ is transfer- $k$-concave in case the solution $\psi$ is additive, $\psi_{i}$ is increasing in $v(i)$ for all $i \in N$ and all $v \in \mathcal{G}^{N}$ and $\psi_{i}(z)=0$ for all $i \in N$. Additionally, $\psi$ is transfer2 -concave if $\psi$ is restrictively additive and $\psi_{i}(z)=0$ for all $i \in N$. The following example illustrates that the nucleolus, a restrictively additive solution, is not transfer-1-concave.

Example 3. Consider the game $v$ where $v(1)=0, v(2)=1, v(3)=0$ and $v(12)=1$, $v(13)=2, v(23)=1$ and $v(N)=6$. The first transfer system is used and only transfers of player 1 are considered. The nucleolus $\nu\left(v+\alpha 1_{1}\right)$ can be computed for different values $\alpha$ and $\nu\left(v+0 \cdot 1_{1}\right)=\nu(v)=\left(1 \frac{3}{4}, 2 \frac{1}{2}, 1 \frac{3}{4}\right), \nu\left(v+\frac{1}{2} \cdot 1_{1}\right)=\left(2,2 \frac{1}{2}, 1 \frac{1}{2}\right)$ and $\nu\left(v+1 \cdot 1_{1}\right)=$ $\left(2 \frac{1}{3}, 2 \frac{1}{3}, 1 \frac{1}{3}\right)$ are obtained. Take $\lambda=\frac{1}{2}$ and strategies $x=0$ and $y=1$ for player 1 , then 
$\nu_{1}\left(\lambda\left(v+x 1_{1}\right)+(1-\lambda)\left(v+y 1_{1}\right)\right)=\nu_{1}\left(\frac{1}{2}\left(v+0 \cdot 1_{1}\right)+\left(1-\frac{1}{2}\right)\left(v+1 \cdot 1_{1}\right)\right)=\nu_{1}\left(v+\frac{1}{2} \cdot 1_{1}\right)=2 \frac{1}{2}$. However $\lambda \nu_{1}\left(v+x 1_{1}\right)+(1-\lambda) \nu_{1}\left(v+y 1_{1}\right)=\frac{1}{2} \nu_{1}\left(v+0 \cdot 1_{1}\right)+\frac{1}{2} \nu_{1}\left(v+2 \cdot 1_{1}\right)=2 \frac{7}{12}$. Since $2 \frac{1}{2} \ngtr 2 \frac{7}{12}$ it follows that the nucleolus is not transfer- 1 -concave.

In Example 3 the cores of the games $v+\alpha 1_{i}$ for $\alpha \in\left\{0, \frac{1}{2}, 1\right\}$ are nonempty and therefore the prenucleolus and nucleolus coincide. This means that both solutions are not transfer-1concave and thus Theorem 4 cannot be used. In the next example we consider the dynamic game $\Gamma=(\mathbf{v}, \mathbf{u})$ where the intertemporal utility function $u_{i}$ is continuous and contained in class $\mathcal{V}$ for all $i \in N$. Payoffs are transferred according to the first transfer system and the solution which is used is restrictively additive. In the example a Nash equilibrium does not exist. This is consistent with the fact that the nucleolus is not transfer-1-concave which thereby causes at least one of the utility functions, $\tilde{u}_{i}$ for $i \in N$, of the associated noncooperative game to be not quasi-concave.

Example 4. Consider the dynamic TU-game $\Gamma=\left(v^{1}, v^{2} ; u_{1}, u_{2}, u_{3}\right)$, the nucleolus $\nu$ and transfer system $k=1$. The game $v^{1}$ is described as $v^{1}(1)=v^{1}(2)=v^{1}(3)=0, v^{1}(12)=$ $v^{1}(23)=1, v^{1}(13)=2$ and $v^{1}(N)=6$ and $v^{2}$ has worths $v^{2}(1)=v^{2}(2)=1, v^{2}(S)=v^{1}(S)$ for all $S \neq\{1\},\{2\}$. Clearly only players 1 and 2 can transfer payoffs between periods 1 and 2, player 3 is just a strategic dummy player. The vectors $\alpha=\left(\alpha^{1}, \alpha^{2}\right) \in \mathcal{S}_{1}$ and $\beta=\left(\beta^{1}, \beta^{2}\right) \in \mathcal{S}_{2}$ contain all possible strategies resulting from transferring payoffs of respectively player 1 and player 2. Moreover, $\alpha^{1}+\alpha^{2}=\beta^{1}+\beta^{2}=1$ and $\alpha^{1}, \beta^{1} \in[0,1]$. The intertemporal utility functions of players 1 and 2 are respectively denoted $u_{1}$ and $u_{2}$ and are contained in the set $\mathcal{V}$. In particular $u_{1}$ and $u_{2}$ are constant discounted utility functions with a discount factor equal to one.

Player 1's utility function $\tilde{u}_{1}(\alpha, \beta)$ of the associated noncooperative game $G_{1}(\Gamma, \nu)$, which equals by definition $u_{1}\left(\nu\left(v^{1}+\alpha^{1} 1_{1}+\beta^{1} 1_{2}\right), \nu\left(v^{2}+\alpha^{2} 1_{1}+\beta^{2} 1_{2}\right)\right)$, is given in Table 2 . It shows for every transfer $\left(\beta^{1}, 1-\beta^{1}\right)$ of player 2 , player 1 's utility.

\begin{tabular}{ll}
\hline value of $\beta^{1}$ & $\tilde{u}_{1}(\alpha, \beta)$ \\
\hline$\beta^{1}=1$ if $\alpha^{1} \in\left[0, \frac{1}{2}\right]$ & $4 \frac{1}{3}+\frac{1}{12} \beta^{1}-\frac{1}{6} \alpha^{1}$ \\
$\beta^{1}=1$ if $\alpha^{1} \in\left[\frac{1}{2}, 1\right]$ & $4 \frac{1}{3}$ \\
$\beta^{1} \in(0,1)$ if $\alpha^{1} \in\left[0, \frac{1}{2} \beta^{1}\right]$ & $4 \frac{1}{3}+\frac{1}{12} \beta^{1}-\frac{1}{6} \alpha^{1}$ \\
$\beta^{1} \in(0,1)$ if $\alpha^{1} \in\left[\frac{1}{2}, \frac{1}{2} \beta^{1}+\frac{1}{2}\right]$ & $4 \frac{1}{3}$ \\
$\beta^{1} \in(0,1)$ if $\alpha^{1}\left[\frac{1}{2} \beta^{1}+\frac{1}{2}, 1\right]$ & $4 \frac{1}{4}+\frac{1}{6} \alpha^{1}-\frac{1}{12} \beta^{1}$ \\
$\beta^{1}=0$ if $\alpha^{1}\left[0, \frac{1}{2}\right]$ & $4 \frac{1}{3}$ \\
$\beta^{1}=0$ if $\alpha^{1} \in\left[\frac{1}{2}, 1\right]$ & $4 \frac{1}{4}+\frac{1}{6} \alpha^{1}-\frac{1}{12} \beta^{1}$ \\
\hline
\end{tabular}

Table 2: Player 1's utility function $\tilde{u}_{1}(\alpha, \beta)$.

Player 2's utility function is given in Table 3. By definition $\tilde{u}_{2}(\alpha, \beta)$ of $G_{1}(\Gamma, \nu)$ is equal to $u_{2}\left(\nu\left(v^{1}+\alpha^{1} 1_{1}+\beta^{1} 1_{2}\right), \nu\left(v^{2}+\alpha^{2} 1_{1}+\beta^{2} 1_{2}\right)\right)$. The table shows for every possible transfer $\left(\alpha^{1}, 1-\alpha^{1}\right)$ of player 1 , player 2 's utility. 


\begin{tabular}{ll}
\hline value of $\alpha^{1}$ & $\tilde{u}_{2}(\alpha, \beta)$ \\
\hline$\alpha^{1} \in\left[0, \frac{1}{2}\right]$ if $\beta^{1} \in\left[0,2 \alpha^{1}\right]$ & $4 \frac{1}{3}$ \\
$\alpha^{1} \in\left[0, \frac{1}{2}\right]$ if $\beta^{1} \in\left[2 \alpha^{1}, 1\right]$ & $4 \frac{1}{3}-\frac{1}{6} \beta^{1}+\frac{1}{3} \alpha^{1}$ \\
$\alpha^{1} \in\left[\frac{1}{2}, 1\right]$ if $\beta^{1} \in\left[0,2 \alpha^{1}-1\right]$ & $4 \frac{1}{2}+\frac{1}{6} \beta^{1}-\frac{1}{3} \alpha^{1}$ \\
$\alpha^{1} \in\left[\frac{1}{2}, 1\right]$ if $\beta^{1} \in\left[2 \alpha^{1}-1,1\right]$ & $4 \frac{1}{3}$ \\
\hline
\end{tabular}

Table 3: Player 2's utility function $\tilde{u}_{2}(\alpha, \beta)$.

Table 4 shows the reaction functions of player 1 and 2 . It contains player 1's best response $\left(\alpha^{1 *}, 1-\alpha^{1 *}\right)$ against any possible transfer $\left(\beta^{1}, 1-\beta^{1}\right)$ of player 2 given the utility function $\tilde{u}_{1}(\alpha, \beta)$ in Table 2 . It also contains player 2 's best response $\left(\beta^{1 *}, 1-\beta^{1 *}\right)$ for every possible transfer $\left(\alpha^{1}, 1-\alpha^{1}\right)$ of player 1 given $\tilde{u}_{2}(\alpha, \beta)$ in Table 3 .

\begin{tabular}{|c|c|c|c|}
\hline \multicolumn{2}{|c|}{ Reaction function player 1} & \multicolumn{2}{|c|}{ Reaction function player 2} \\
\hline value of $\beta^{1}$ & best response $\alpha^{1 *}$ & value of $\alpha^{1}$ & best response $\beta^{1} *$ \\
\hline $\begin{array}{l}\beta^{1} \in\left[0, \frac{1}{2}\right] \\
\beta^{1} \in\left[\frac{1}{2}, 1\right]\end{array}$ & $\begin{array}{l}\alpha^{1 *}=1 \\
\alpha^{1 *}=0\end{array}$ & $\begin{array}{l}\alpha^{1} \in\left[0, \frac{1}{2}\right] \\
\alpha^{1} \in\left[\frac{1}{2}, 1\right]\end{array}$ & $\begin{array}{l}\beta^{1 *} \in\left[0,2 \alpha^{1}\right] \\
\beta^{1 *} \in\left[2 \alpha^{1}-1,1\right]\end{array}$ \\
\hline
\end{tabular}

Table 4: Player 1's and 2's reaction functions.

Player 1 and 2's reaction functions are displayed in Figure 1, which shows no overlap of best response sets of both players. Hence, no Nash equilibrium exists.

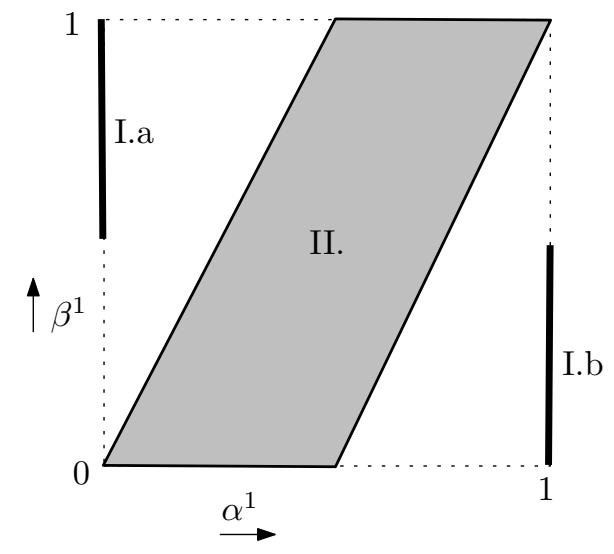

Figure 1: Best response sets of players 1 and 2. Player 1's best response set consists of parts I.a and I.b, player 2's best response set consists of part II.

The notion of Nash equilibrium has a number of limitations. In the next section one of these shortcomings is considered.

\section{Pareto optimality}

A drawback of Nash equilibrium is that it is not immune to coalitional deviations. As a result Pareto optimality of a Nash equilibrium is not guaranteed. In this section Nash 
equilibria in weakly dominant strategies are considered where players transfer as much payoff as possible to time period 1. It is shown in which cases these equilibria are weakly and strongly Pareto optimal. A strategy profile $\alpha^{*} \in \mathcal{S}_{N}$ is weakly Pareto optimal if there exists no strategy profile $\alpha \in \mathcal{S}_{N}$ such that $\tilde{u}_{i}(\alpha)>\tilde{u}_{i}\left(\alpha^{*}\right)$ for all $i \in N$. A strategy profile $\alpha^{*} \in \mathcal{S}_{N}$ is strongly Pareto optimal if there exists no strategy profile $\alpha \in \mathcal{S}_{N}$ such that $\tilde{u}_{i}(\alpha) \geq \tilde{u}_{i}\left(\alpha^{*}\right)$ for all $i \in N$ with at least one strict inequality.

In the next theorem the noncooperative game $G_{2}(\Gamma, \psi)$ is considered where $\psi$ is restrictively additive and $\psi_{i}(z)=0$ for all $i \in N$.

Theorem 5 Let $\Gamma=(\mathbf{v}, \mathbf{u})$ be a dynamic game, let $v^{t} \in \mathcal{G}^{N}$ be weakly superadditive for all $t \in\{1, \ldots, T\}$, let $\psi$ be restrictively additive and let $\psi_{i}(z)=0$ for all $i \in N$.

(i) If $u_{i} \in \mathcal{V}$ for all $i \in N$, then the strategy profile that contains for every $i \in N$ strategy $\left(\sum_{t=2}^{T} v^{t}(i),-v^{2}(i), \ldots,-v^{T}(i)\right)$ is weakly Pareto optimal in $G_{2}(\Gamma, \psi)$.

(ii) If $u_{i} \in \overline{\mathcal{V}}$ for all $i \in N$, then the strategy profile that contains for every $i \in N$ strategy $\left(\sum_{t=2}^{T} v^{t}(i),-v^{2}(i), \ldots,-v^{T}(i)\right)$ is strongly Pareto optimal in $G_{2}(\Gamma, \psi)$.

Proof. (i) Let $u_{i} \in \mathcal{V}$ for all $i \in N$. Since $\psi$ is restrictively additive and for all $i \in N$ it holds that $\psi_{i}(z)=0$ and $u_{i} \in \mathcal{V}$ it follows from Theorem $3(\mathrm{i})$ that $\left(\sum_{t=2}^{T} v^{t}(i),-v^{2}(i), \ldots,-v^{T}(i)\right)$ for all $i \in N$ is a Nash equilibrium in $G_{2}(\Gamma, \psi)$. Moreover, because $u_{i} \in \mathcal{V}$ this Nash equilibrium is weakly Pareto optimal, since for every player $i \in N$ it holds that strategy $\left(\sum_{t=2}^{T} v^{t}(i),-v^{2}(i), \ldots,-v^{T}(i)\right)$ generates player $i$ 's maximum utility independent of other players strategies.

(ii) Let $u_{i} \in \overline{\mathcal{V}}$ for all $i \in N$. Since $\psi$ is restrictively additive and for all $i \in N$ it holds that $\psi_{i}(z)=0$ and $u_{i} \in \overline{\mathcal{V}}$ it follows from Theorem 3(ii) that $\left(\sum_{t=2}^{T} v^{t}(i),-v^{2}(i), \ldots,-v^{T}(i)\right)$ for all $i \in N$ is a unique Nash equilibrium in $G_{2}(\Gamma, \psi)$. Because $u_{i} \in \overline{\mathcal{V}}$ for every player $i \in N$ it holds that strategy $\left(\sum_{t=2}^{T} v^{t}(i),-v^{2}(i), \ldots,-v^{T}(i)\right)$ yields player $i$ his strictly highest level of utility independent of other players strategies, which means that this Nash equilibrium is strongly Pareto optimal.

The nucleolus as well as the Shapley value are restrictively additive and satisfy the conditions of Theorem 5. In the case where we consider noncooperative game $G_{1}(\Gamma, \psi)$ it is not clear if Nash equilibria where as much as possible is transferred to the first time period are Pareto optimal. Another interesting point to consider is whether the above mentioned Nash equilibria are strong Nash equilibria. A strong Nash equilibrium is immune to all coalitional deviations and is defined as a strategy combination $\alpha^{*} \in \mathcal{S}_{N}$, such that for all coalitions $M \subseteq N$ and all $\alpha_{M} \in \mathcal{S}_{M}$ there exists a player $i \in M$ such that $\tilde{u}_{i}\left(\alpha_{M}, \alpha_{N \backslash M}^{*}\right)<\tilde{u}_{i}\left(\alpha^{*}\right)$. With respect to strong Nash equilibria we did not find a general result.

\section{Concluding remarks}

In this paper it is assumed that players can only make individual intertemporal transfers. Kranich et al. (2000) mention briefly the influence of an aggregate transfer, which they 
define as a transfer of payoffs of a coalition of players, $S$ with $|S| \geq 2$, which increases the worth of $S$ at time $t, v^{t}(S)$, and decreases the worth of $S$ at time $t^{\prime}, v^{t^{\prime}}(S)$. Further research could look at the role of aggregate transfers in dynamic games. Another limitation of the model is its impossibility to transfer more payoff than the worth of the individual coalition. It is only possible for an individual to borrow from his own future. However, in real life situations an individual can take out a mortgage and go bankrupt. Other research could look at a possible extension of the model developed by Filar and Petrosjan (2000) to include transfers.

\section{References}

Debreu, G. (1952): "A Social Equilibrium Existence Theorem," Proceedings of the National Academy of Sciences of the U.S.A., 38, 386-393.

Filar, J.A., and L.A. Petrosjan (2000): "Dynamic Cooperative Games," International Game Theory Review, 2, 47-65.

Fishburn, P.C., and A. Rubinstein (1982): "Time Preference," International Economic Review, 23, 677-694.

Kranich, L., A. Perea, and H. Peters (2000): "Dynamic Cooperative Games," Unpublished Mimeo.

Kranich, L., A. Perea, and H. Peters (2005): "Core Concepts for Dynamic TU Games," International Game Theory Review, 7, 43-61.

Peleg, B., and P. Sudhölter (2003): Introduction to the Theory of Cooperative Games. Boston: Kluwer Academic Publishers.

Schmeidler, D. (1969): "The Nucleolus of a Characteristic Function Game," SIAM Journal on Applied Mathematics, 17, 1163-1170.

Shapley, L.S. (1953): "A Value for n-Person Games," in: A.W. Tucker, H.W. Kuhn (eds.), Contributions to the Theory of Games II, 307-317. Princeton, New Jersey: Princeton University Press. 